\title{
Short-Term Load Forecasting of Active and Reactive Powers in Small Scales using LASSO-Integrated Nonlinear Autoregressive Neural Networks
}

\author{
Guanchen Zhang, G. Gary Wang, Hassan Farhangi, and Ali Palizban
}

\begin{abstract}
Short-term load forecasting (STLF) is important for power system planning and optimization, especially in the dynamic environment of smart grid. Traditional load forecasting is implemented at substation levels to predict the upcoming active power and optimal system settings. In more advanced smart grid applications, e.g. the Volt-VAR Control, small-scale load forecasting opens up new opportunities in coordinating distributed resources such as distributed generation (DG) with utilities' efficiency missions. This paper proposes a STLF approach for small residential blocks with $\mathbf{1 0 - 1 2}$ households. The Nonlinear Autoregressive Neural Network (NAR-NN) is employed to predict hour-ahead active $(P)$ and reactive $(Q)$ powers with a moving window of training data. The regressor shrinkage technique, LASSO, is used to improve the selection of the regressors in the NAR-NN model by removing insignificant input features. The results show the forecasting performance could be enhanced by $20 \%$ comparing to feed-forward Artificial Neural Networks (ANNs). The improvement in forecasting both $P \& Q$ could accommodate new smart grid applications in small scales.
\end{abstract}

Index Terms-Short-term load forecasting, nonlinear autoregressive neural network, regression shrinkage, power system planning, smart grid.

\section{INTRODUCTION}

Smart grid technologies and applications, built on top of advanced communication architectures, take advantage of coordinative and efficient management of various grid assets in generation, transmission and distribution systems. As more decentralized factors become more effective in the smart grid, more dynamic and robust solutions to system efficiency are necessary. For example, the distributed generation (DG) has been integrated into the Volt-VAR Control (VVC) system in distribution networks [1]. Electric vehicles (EVs) could also serve as DGs in VVC systems to compensate for reactive power [2]. The management algorithms of such applications require the short-term load forecast (STLF) of active and reactive powers in smaller scales to dispatch resources and predict optimal set points for transformers and capacitor banks (CBs). STLF is also important in Demand Response (DR) in evaluating and selecting the DR solutions [3]. Other

Guanchen Zhang (gza10@sfu.ca) and G. Gary Wang are with the School of Mechatronic Systems Engineering, Simon Fraser University, Surrey, BC, Canada.

Hassan Farhangi and Ali Palizban are with British Columbia Institute of Technology, Burnaby, BC, Canada.

Manuscript prepared on February 2, 2016. Revised on October 2, 2016. applications of STLF include the predictive control of heating and cooling in homes and buildings [4].

STFL is a critical step in designing the optimal solutions in the dynamic environment of smart grid. Traditional STFL focuses on large-scales such as substations in which data are more smooth [5]. STFL in small scales (individual houses or small community) typically suffer from behavioral randomness that result in consumption data with stochastic patterns.

In this paper, the STLF on the scale of 10-12 households is studied. The objective is to improve the performance of STLF on such small scale to facilitate applications such as the Volt-VAR Optimization engine in [2]. Other applications that demand forecasting on such small scale could also utilize the forecasting approach in this paper. The Nonlinear Autoregressive Neural Network (NAR-NN) and NAR-NN with exogenous inputs (NARX-NN) are employed to predict the hour-ahead active and reactive power consumption. The overfitting issue in NN models is handled by integrating the Least Absolute Shrinkage and Selection Operator (LASSO) technique to eliminate insignificant inputs of the NN model. The switch between NAR-NN and NARX-NN depends on the LASSO selection. That is, if no exogenous input (e.g. temperature) is selected, the NAR-NN will be used; otherwise, the NARX-NN will be used. The NAR(X)-NN forecasting model outperforms the simple ANN forecasting model with no input filtering by $20 \%$ error reduction. The NAR(X)-NN model is proved to be applicable to both active $(\mathrm{P})$ and reactive (Q) power forecasting, and the $\mathrm{Q}$ forecasting is independent of $\mathrm{P}$. The main contributions of this paper include:

- A STLF model based on the ANN integrated with regressor-shrinkage technique (LINAR(X)-NN)

- Shrinkage of insignificant regressors and optimization of the training window for the $\operatorname{LINAR}(\mathrm{X})-\mathrm{NN}$

- Application in ultra small scales of single houses and 10-12 house aggregation

- Independent forecasting of reactive power

The remainder of the paper is organized as the following. Section II briefly reviews related works. Section III explains the proposed forecasting model. Section IV demonstrates the forecasting results on various scales, from single house to 1012 houses. Section V concludes this paper. 


\section{RELATED WORKS}

The STFL falls into the category of time-series forecasting. Classical approaches of time-series forecasting based on linear models include the Autoregressive Moving Average (ARMA) [6], Autoregressive Integrated Moving Average (ARIMA) [7], Generalized Autoregressive Conditional Heteroskedastic $(\mathrm{GARCH})$ [8], and the variants of these models. Nonlinear techniques feature the Artificial Neural Network (ANN) and its variants (e.g. Multi-Layer Perceptron (MLP), Radial Base Function (RBF), Self-Organizing Map (SOM)) [9] and Support Vector Regression (SVR). The ANN has been proved to be an effective tool in STLF, particularly in small scales [10]. Although the SVR has good performance in large scales, it is outperformed by simple linear models in small scales [11].

Generally, most existing models have comparable performance at large scales, e.g. substation, and the advantages of certain models depend on applications and complexity. When dealing with single houses or 10-12 houses, existing models may not generate useful accuracy. In [11], a relation between the number of houses and forecasting error is illustrated as an Exponential curve with average $60 \%$ error on the single-house level and less than $10 \%$ on over 100-house scales.

The NARX-NN model is proved to be able to predict chaotic time series [12]. The application of the NARX-NN on STLF is tested in [13]-[15], in which the authors forecast megawatt (MW) level loads in large scales. For small scales, however, the NARX-NN is not widely tested. One of the challenges in ANN-based forecast is the overfitting problem, which is also a problem in general ANN models. The problem becomes more challenging if a large amount of input features are used, and no explicit rules of input selection are applied. Many forecasting models incorporate exogenous data such as weather and intuition-based selection of recurrent orders (e.g. load at the same hour of previous days). However, general selection of internal and external inputs may not apply to every scenario. Li et al. in [16] apply the LASSO technique in the AR model to forecast the load of single houses. The AR order in their model is adjusted by the output of the LASSO. The average error for single house is below $25 \%$, and for the 10-house case the error is around $10 \%$. Hashmi et al. in [14] attempt to solve the overfitting problem by finding the optimal number of layer delays in the NARX-NN; however, the efficient use of exogenous inputs should also be considered.

\section{PROBLEM FORMULATION}

\section{A. $N A R(X)-N N$}

Comparing with the feed-forward multi-layer neural network, the NAR-NN additionally considers recurrent and delayed input components that form the autoregressive model in (1). Because of the recurrence, the NAR model could be implemented in either open or closed loop.

$$
\hat{y}_{t}=f\left(y_{t-1}, y_{t-2}, \ldots, y_{t-n_{y}}\right) \text {, }
$$

where $\hat{y}_{t}$ is the predicted output at time instance $t, y_{t-i}$ is the true output at $t-i$, and $n_{y}$ is the number of layer delays.
The NARX-NN allows exogenous inputs and alters (1) into (2), where $n_{x}$ denotes the number of input delays for the exogenous input $x$.

$$
\begin{array}{r}
\hat{y}_{t}=f\left(y_{t-1}, y_{t-2}, \ldots, y_{t-n_{y}},\right. \\
\left.x_{t}, x_{t-1}, \ldots, x_{t-n_{x}}\right) .
\end{array}
$$

Fig. 1 illustrates the structure of the NAR(X)-NN model. The NAR-NN neglects the external inputs $x$. In case of openloop prediction, the output $\hat{y}_{t}$ is not fed back for predicting the next time instance $t+1$, but the known or measured true output $y_{t}$ at time $t$. Note in this paper, $y_{t}$ is equivalent to $y(t)$.

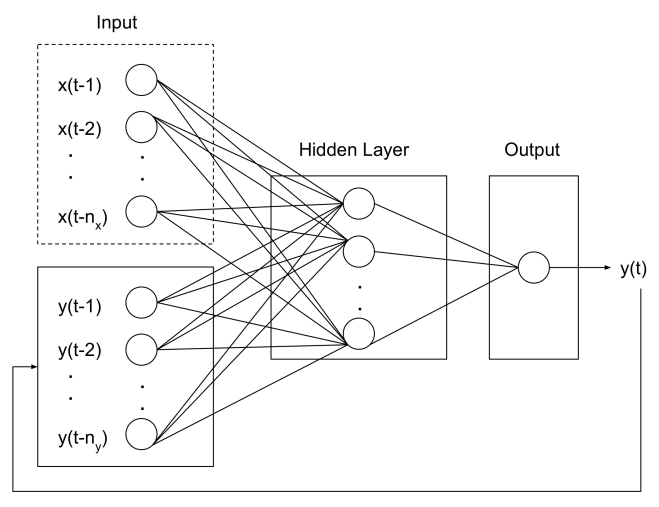

Fig. 1: NARX-NN structure

\section{B. LASSO}

1) Background: The LASSO is a shrinkage regression technique with L1 norm regulation for linear regression [17]. The idea is to reduce the mean square error (MSE) in the ordinary least square (OLS) problem by slightly increasing the bias and lowering the variance in the estimated parameters of the new regularized OLS model. This means the coefficients of some of the regressors could be reduced to zeros. The LASSO in this paper takes inputs as the potential endogenous and exogenous features that relate to the load forecasting model. The optimal parameters in the LASSO correspond to the minimal MSE. By applying the LASSO process, features that are not strongly correlated to load forecasting, although intuitively they seem to be, can be eliminated. The detailed definition of LASSO is described in (3) ${ }^{1}$.

$$
\min _{\beta_{0}, \beta}\left(\frac{1}{2 N} \Sigma_{t=1}^{N}\left(y_{t}-\beta_{0}-x_{t} \beta\right)^{2}+\lambda \Sigma_{j=1}^{p}\left|\beta_{j}\right|\right),
$$

where $N$ denotes the number of observations, $y_{t}$ is the response at time $t, \beta_{0}$ is the interception, $\beta$ is the vector of regression coefficients, $x_{t}$ is vector of regressors at $t, \lambda$ is the regulation parameter, and $p$ is the number of regressors.

The purpose of the LASSO is to, on the top level, select the related inputs in a generic way. This is particularly useful when dealing with single or small number of households for which

\footnotetext{
${ }^{1}$ Adapted from https://www.mathworks.com/help/stats/lasso.html
} 
the correlated inputs are different and non-consistent across different time windows. The layer and input delays in (1) and (2) are also nontrivial, as the future load may correlate to any past time instance and differ in households. Another reason for using LASSO is to mitigate the overfitting problem in the NN when an overwhelming amount of inputs are injected (e.g. high recurrence order and consecutive delays).

2) Input Candidates: The potential exogenous inputs in this paper include the ambient temperature in Celsius, the timestamps of smart meter readings, whether it is workday or holiday, and the day of week. The endogenous inputs consist of previous smart meter readings from one hour ahead to 48 hours ahead. The LASSO selects the inputs for the NAR(X)$\mathrm{NN}$ from these candidates, and informs whether a NAR or NARX NN should be used.

\section{C. $N A R(X)-N N$ Setup}

To further improve the performance of the NAR(X)-NN, the Bayesian regulation backpropagation algorithm is used to train the NN by further eliminating the unnecessary weights and biases in the lower level of the NAR(X)-NN. All the input data are normalized to $[-1,1]$ to accommodate the Bayesian regulation algorithm.

After the training of the $\mathrm{NAR}(\mathrm{X})-\mathrm{NN}$ with historical data, the training data are altered in a first-in-first-out (FIFO) fashion (Fig. 2). The NAR(X)-NN is sequentially trained by the new set of training data rather than being directly applied to the new forecasting set (open-loop forecasting). This allows improved overall performance of the NAR(X)-NN by capturing shortterm variations, and in addition, eases the requirement of large amount of historical data needed to train the NAR(X)-NN.

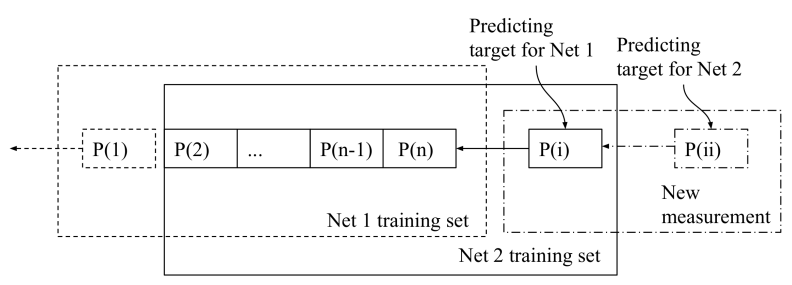

Fig. 2: Receding training window for hour-ahead NAR(X)-NN

\section{Model Evaluation}

Unlike the forecasting on large scales in which the mean absolute percent of error (MAPE) is widely used, the forecasting on small scales in this paper is evaluated based on the normalized root mean squared error (NRMSE, described in (4)). The reason is that the MAPE may become unreliable when the consumption data are small or close to zero, which is common in small scales; whereas the NRMSE is not affected by such problem [11].

$$
\begin{aligned}
N R M S E(Y, \hat{Y}) & =\frac{R M S E(Y, \hat{Y})}{\|Y\|_{2}} \\
& =\frac{\sqrt{\frac{1}{N} \Sigma_{t=1}^{N}\left(y_{t}-\hat{y}_{t}\right)^{2}}}{\sqrt{\frac{1}{N} \Sigma_{t=1}^{N} y_{t}^{2}}},
\end{aligned}
$$

where $Y$ is the actual response, $\hat{Y}$ is the prediction, $\|\cdot\|_{2}$ is the L2 norm, $N$ is the number of observations, $y_{t}$ is the consumption at $t$, and $\hat{y}_{t}$ is the predicted consumption at $t$.

\section{RESULTS}

\section{A. Input Data}

The consumption data of individual houses are obtained from the Pecan Street Database ${ }^{2}$. Data from real households are available in various frequencies, and the hourly data are used in this paper. The corresponding weather data are obtained from Weather Underground ${ }^{3}$. The amount of historical data for training the NAR(X)-NN is selected based on performing LASSO on varying lengths of training window (details explained in Section F).

\section{B. Forecasting Scenarios}

To demonstrate the robustness of our model, data with less temperature dependence are tested to show the effectiveness of the LASSO and the integration with $\mathrm{NAR}(\mathrm{X})-\mathrm{NN}$. In particular, we test the model in moderate season (e.g. spring) for open-loop (hour-ahead) cases of both single and 10-12 houses. The open-loop scenario requires knowing the actual consumption, $y_{t}$, prior to solving for $\hat{y}_{t+1}$.

\section{Open-Loop Test on 10-House Aggregation}

To construct the loads that possibly represent small residential blocks, we use data from 10 houses in the Pecan Street database and add them up as an aggregation for load forecasting. The LASSO finds three related inputs: the average load over the previous 24 hours $\left(\bar{P}_{24}\right)$, and the instantaneous load at one hour and 24 hours ago $\left(P_{1}, P_{24}\right) . \bar{P}_{24}$ is regarded as external. One thing to note in this paper is that the recursive order, or layer delay, is not a continuous series as in other papers. Instead, the layer delays, in this case, are discrete values as 1 and 24 .

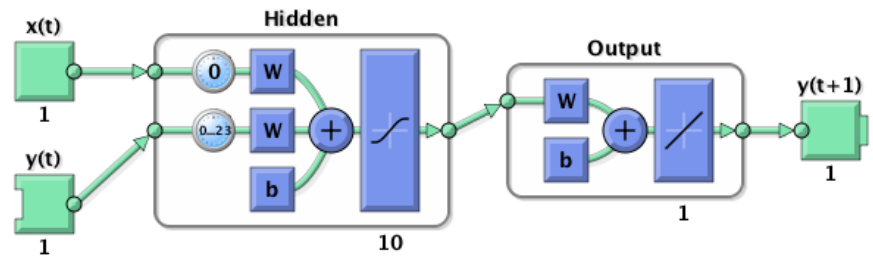

Fig. 3: Open-loop NARX-NN structure for hour-ahead prediction

A month of historical data are first used to train the NARXNN. The performance is evaluated based on the forecasting error on a new set of data that succeeds the historical data. The NRMSE on the new set of data is 0.16 . The error correlation plot is shown in Fig. 4, in which the insignificant lags are within the $95 \%$ confidence interval (CI).

Fig. 5 shows the hour-ahead forecasted consumption on March 25-26, 2014. The upper and lower bound of the 95\% CI

\footnotetext{
${ }^{2} \mathrm{http}: / / \mathrm{www} \cdot$ pecanstreet.org/about/

${ }^{3} \mathrm{http}: / /$ www.wunderground.com/
} 


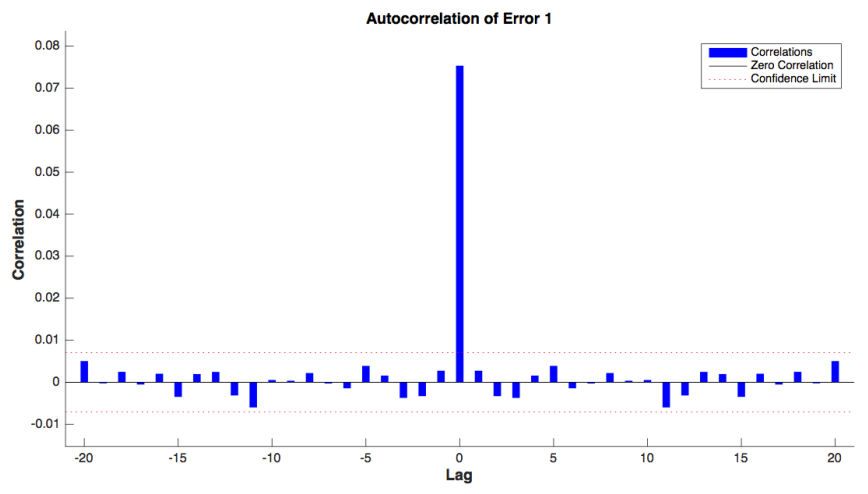

Fig. 4: Error autocorrelation in the NAR(X)-NN

are obtained based on the 95 percentile and 5 percentile of the forecast errors (Gaussian distribution) in the test period. The spring period is chosen to emulate the worst-case scenarios when exogenous inputs are weakly related to consumption. On the other hand, for example in summer, A/C loads may cause the overall consumption more predictable.

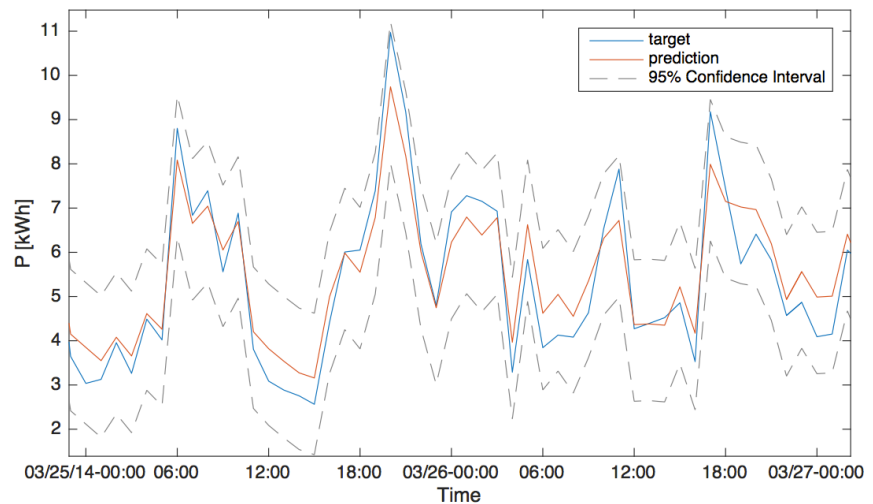

Fig. 5: Predicted active consumption with 95\% CI

The same NARX-NN is applied on the reactive power forecasting. The additional selected input by LASSO is the load at 48 hours ago $\left(P_{48}\right)$. The overall NRMSE is 0.19 , indicating that the NARX-NN approach is applicable to both $\mathrm{P}$ and $\mathrm{Q}$ forecast. The result for the same time period in Fig. 5 is illustrated in Fig. 6.

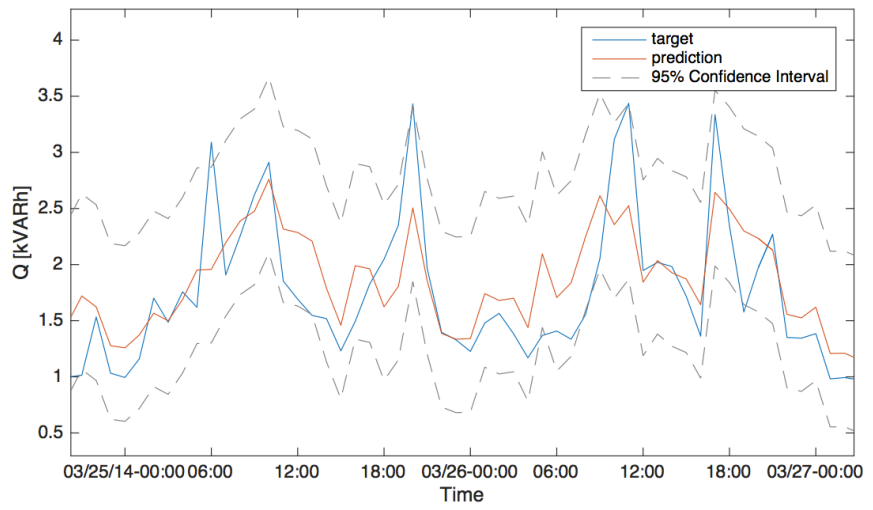

Fig. 6: Predicted reactive consumption with 95\% CI

\section{Open-Loop Test on Single House}

The NAR(X)-NN model is tested on the consumption data of a single house to examine the possibility of predicting single-house consumption. The result of hour-ahead prediction with three weeks of training data is presented in Fig. 7. The resulted NRMSE is 0.26 . In this case, no exogenous input is selected, and the layer delays are on the first and 24th orders. Note that the Pecan Street Data for each house do not contain all the loads, so the forecast error is likely larger than that on the full data of a typical house.

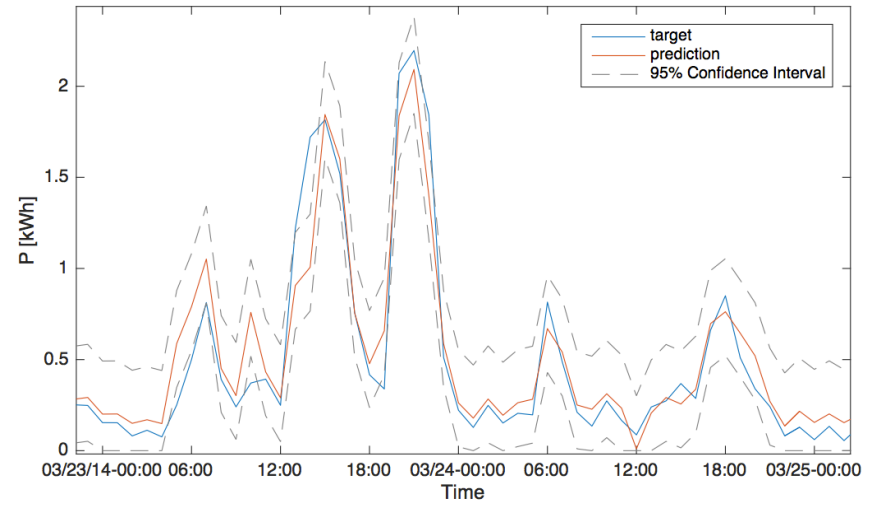

Fig. 7: Predicted active consumption for a single house

\section{E. Performance Comparison}

In this section, the performance of the $\mathrm{NAR}(\mathrm{X})-\mathrm{NN}$ is compared with a feed-forward ANN and the NAR(X)-NN without LASSO. In the ANN model and NAR(X)-NN without LASSO, all the most common inputs are selected. As seen in Table I, the LASSO-integrated NAR(X)-NN, or LINAR(X)$\mathrm{NN}$, outperforms the other two models.

TABLE I: Forecasting Model Comparison for 10-House Aggregation

\begin{tabular}{llll}
\hline & ANN & NAR(X)-NN & LINAR(X)-NN \\
\hline \hline Exogenous & All exgo. in- & All exog. in- & $P_{24}$ \\
inputs & puts & puts & \\
Endogenous & $P_{1}, P_{2}, P_{12}$, & $P_{1}, P_{2}, P_{12}$, & $P_{1}, P_{24}$ \\
inputs & $P_{24}, P_{36}, P_{48}$, & $P_{24}, P_{36}, P_{48}$, & \\
& $\bar{P}_{w e e k}$ & $\bar{P}_{w e e k}$ & \\
NRMSE & 0.34 & 0.26 & 0.16 \\
\hline
\end{tabular}

\section{F. Sensitivity Analysis and Training Data Selection}

Because short training periods are used in this paper to capture the short-term load variance, it is necessary to find the optimal and dynamic length of training data so that the best performance could be achieved. Long training window (e.g. multiple years) is slow to adapt the variance in the new data, and therefore not considered in this paper.

The approach used in this paper to find the optimal training window is implemented by performing the LASSO on backward-expanding training windows prior to the forecasting window. For example, if the target is to forecast the load at $3 / 20 / 2014$ 1:00, the data before this instance backward until 
the LASSO outputs start to change are used as the training set. If a month of data is selected for training, then the training period is from 2/20/2014 0:00 to 3/19/2014 23:00. The default training length is selected as 30 days. In the context of openloop forecasting, the training window is dynamically updated.

Another factor that affects the forecasting performance is the outputs of the LASSO (i.e. the inputs into the NAR(X)$\mathrm{NN})$. The selected inputs from LASSO with small coefficients are further filtered out (Fig. 8 bottom). This eliminates the need for finding the optimal number of hidden layers, which involves computation-heavy iterations.
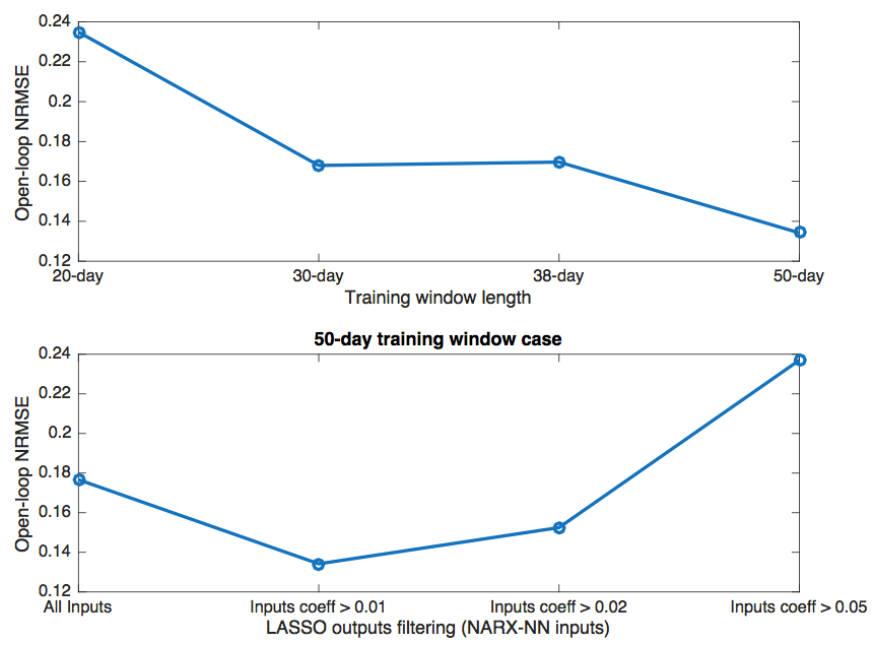

Fig. 8: Sensitivity analysis

Based on Fig. 8, it is found out that performing LASSO on backward-expanding training windows can find the local optima (at 30 days). Increasing the default training window length can find the optimal training window corresponding to the global minima if more data are available (in which case the default training window length can be set as $\geq 50$ days). An example of when the global optima will be located will be during seasonal transition. In addition, removing the inputs with very small LASSO coefficients can also improve the forecasting performance for a fixed number of hidden layers in the NARX-NN (10 layers).

\section{CONCLUSION}

The STLF problem in ultra-small scales is tackled by using the $\mathrm{NAR}(\mathrm{X})-\mathrm{NN}$ model integrated with LASSO regressor selection ( $\operatorname{LINAR}(\mathrm{X})-\mathrm{NN})$. The variance and randomness in small-scale consumption data are handled by the NAR(X)-NN that is capable of modeling dynamic time series. The performance of the $\mathrm{NAR}(\mathrm{X})-\mathrm{NN}$ is further improved by shrinking the NAR(X)-NN inputs and filtering out unnecessary ones which may adversely affect the complexity of the neural network. In the hour-ahead open-loop forecasting context, the LINAR(X)-NN is dynamically re-trained with new measurement to capture short-term variations. The results show that close to $10 \%$ NRMSE can be achieved for the scale of 10-12 houses, whereas the conventional feedforward ANN generates $>30 \%$ NRMSE. Better performance could be possible if more training data are used. The $\operatorname{LINAR}(\mathrm{X})-\mathrm{NN}$ forecasting model could be applied in small-scale utility applications such as planning and dispatching DGs in VVC, as well as in other predictive applications in smart grids concerning energy efficiency and conservation.

\section{REFERENCES}

[1] J. Barr and R. Majumder, "Integration of distributed generation in the volt/var management system for active distribution networks," IEEE Transactions on Smart Grid, vol. 6, no. 2, pp. 576-586, 2015.

[2] M. Manbachi, H. Farhangi, A. Palizban, and S. Arzanpour, "A novel volt-var optimization engine for smart distribution networks utilizing vehicle to grid dispatch," International Journal of Electrical Power \& Energy Systems, vol. 74, pp. 238-251, 2016.

[3] P. B. Lub, L. D. Michel, P. Friedland, C. Guan, and Y. Wang, "Load forecasting and demand response," in Power and Energy Society General Meeting, 2010 IEEE. IEEE, 2010.

[4] W. J. Cole, K. M. Powell, E. T. Hale, and T. F. Edgar, "Reduced-order residential home modeling for model predictive control," Energy and Buildings, vol. 74, pp. 69-77, 2014.

[5] Y. Chen, P. B. Luh, and S. J. Rourke, "Short-term load forecasting: similar day-based wavelet neural networks," in Intelligent Control and Automation, 2008. WCICA 2008. 7th World Congress on. IEEE, 2008, pp. 3353-3358.

[6] S.-J. Huang and K.-R. Shih, "Short-term load forecasting via arma model identification including non-gaussian process considerations," IEEE Transactions on Power Systems, vol. 18, no. 2, pp. 673-679, 2003.

[7] C.-M. Lee and C.-N. Ko, "Short-term load forecasting using lifting scheme and arima models," Expert Systems with Applications, vol. 38, no. 5, pp. 5902-5911, 2011.

[8] R. C. Garcia, J. Contreras, M. Van Akkeren, and J. B. C. Garcia, "A garch forecasting model to predict day-ahead electricity prices," IEEE transactions on power systems, vol. 20, no. 2, pp. 867-874, 2005.

[9] L. Hernandez, C. Baladrón, J. M. Aguiar, B. Carro, A. J. SanchezEsguevillas, and J. Lloret, "Short-term load forecasting for microgrids based on artificial neural networks," Energies, vol. 6, no. 3, pp. 1385 1408, 2013

[10] J. Cavallo, A. Marinescu, I. Dusparic, and S. Clarke, "Evaluation of forecasting methods for very small-scale networks," in International Workshop on Data Analytics for Renewable Energy Integration. Springer, 2015, pp. 56-75.

[11] T. K. Wijaya, M. Vasirani, S. Humeau, and K. Aberer, "Clusterbased aggregate forecasting for residential electricity demand using smart meter data," in Big Data (Big Data), 2015 IEEE International Conference on. IEEE, 2015, pp. 879-887.

[12] E. Diaconescu, "The use of narx neural networks to predict chaotic time series," Wseas Transactions on computer research, vol. 3, no. 3, pp. 182-191, 2008.

[13] L. C. M. de Andrade, M. Oleskovicz, A. Q. Santos, D. V. Coury, and R. A. S. Fernandes, "Very short-term load forecasting based on narx recurrent neural networks," in 2014 IEEE PES General MeetingConference \& Exposition. IEEE, 2014, pp. 1-5.

[14] M. U. Hashmi, V. Arora, and J. G. Priolkar, "Hourly electric load forecasting using nonlinear autoregressive with exogenous (narx) based neural network for the state of goa, india," in Industrial Instrumentation and Control (ICIC), 2015 International Conference on. IEEE, 2015, pp. $1418-1423$.

[15] S. Muralidharan, A. Roy, and N. Saxena, "Stochastic hourly load forecasting for smart grids in korea using narx model," in 2014 International Conference on Information and Communication Technology Convergence (ICTC). IEEE, 2014, pp. 167-172.

[16] P. Li, B. Zhang, Y. Weng, and R. Rajagopal, "Autoregressive model for individual consumption data - lasso selection and significance test," 2015.

[17] J. Friedman, T. Hastie, and R. Tibshirani, The elements of statistical learning. Springer series in statistics Springer, Berlin, 2001, vol. 1. 